\title{
ILUSTRAÇÃO DE CARACTERES MICROSCÓPICOS DE DROGAS VEGETAIS PARA O CONTROLE DE QUALIDADE FARMACOGNÓSTICO. IV. JABORANDI (Pilocarpus pennatifolius LEM., RUTACEAE)
}

\section{ILLUSTRATION OF VEGETAL DRUG MICROSCOPIC CHARACTERS FOR THE PHARMACOGNOSTIC QUALITY CONTROL. IV. JABORANDI (Pilocarpus pennatifolius LEM., RUTACEAE)}

\author{
DUARTE, M. do R. ${ }^{1}$; GOLAMBIUK, G. ${ }^{2}$; COSTA, R. E. ${ }^{2}$ \\ ${ }^{1}$ Laboratório de Farmacognosia, Departamento de Farmácia, Universidade Federal do Paraná \\ (UFPR), Av. Pref. Lothário Meissner, 632, Jardim Botânico, 80210-170, Curitiba, PR, marciard@ufpr. \\ br \\ ${ }^{2}$ Curso de Farmácia, UFPR \\ REC:05/09 AC:06/09
}

\begin{abstract}
RESUMO
O presente trabalho faz parte de uma série de publicações deste periódico, que ilustram os caracteres microscópicos de drogas vegetais, como recurso didático e complementar à descrição anatômica. A espécie em questão é Pilocarpus pennatifolius Lem., uma das várias Rutaceae conhecidas como jaborandi. A parte usada é a folha composta ou as subunidades, denominadas de folíolos, que são ricos em alcaloides imidazólicos, principalmente pilocarpina. Amostras de folhas adultas foram coletadas de exemplares identificados no Jardim Botânico Municipal de Curitiba. O material foi fixado em FAA, seccionado à mão livre ou emblocado em glicometacrilato, seccionado em micrótomo e corado. As ilustrações mostram folha hipoestomática, estômatos anomocíticos, cutícula estriada, tricomas tectores unicelulares, tricomas glandulares capitados pluricelulares, mesofilo dorsiventral, nervura central e peciólulo biconvexos em secção transversal, drusas de oxalato de cálcio e cavidades secretoras.
\end{abstract}

Palavras-chave: Anatomia, droga vegetal, ilustração, microscopia.

\begin{abstract}
This work integrates a series of this journal, which displays microscopic characters of vegetal drugs, as an applicable resource that complements anatomical description. The species herein is Pilocarpus pennatifolius Lem., one among various Rutaceae popularly known as jaborandi. The vegetal drug consists of compound leaves or subunits, called leaflets, which are rich in imidazole alkaloids, mainly pilocarpine. Samples of mature leaves were collected from taxonomically identified specimens at the Jardim Botânico Municipal de Curitiba. The material was fixed in FAA, free-hand sectioned or embedded in glycol-methacrylate, sectioned by microtome and stained. The illustrations show hypostomatic leaf, anomocytic stomata, striate cuticle, unicellular non-glandular trichomes, multicellular capitate trichomes, dorsiventral mesophyll, midrid and petiolule biconvex in transection, calcium oxalate druses and secretory cavities.
\end{abstract}

Keywords: Anatomy, illustration, microscopy, vegetal drug. 


\section{INTRODUÇÃO}

Para a realização do controle de qualidade farmacognóstico, a etapa inicial que possibilita a confirmação da identidade de uma droga vegetal compreende as identificações macroscópica e microscópica. A primeira refere-se à investigação morfológica externa, a qual descreve a forma e o tamanho da parte usada da espécie medicinal. A identificação microscópica trata dos caracteres anatômicos, que mostram a organização de células, tecidos e sistemas que compõem a droga. Ambas as análises consistem de descrições detalhadas, que são complementadas por meio de ilustrações correspondentes. Uma série de artigos vem sendo publicada neste periódico (DUARTE, 2007a; 2007b; DUARTE; GOLAMBIUK, 2008), a fim de apresentar ilustrações que representem fielmente a estrutura da droga vegetal e que facilitem o entendimento das descrições morfológicas para a confirmação da identidade da mesma.

Sob essa perspectiva e enfocando representantes da flora brasileira, o presente trabalho destaca Pilocarpus pennatifolius Lem., uma das espécies de Rutaceae denominadas comumente de jaborandi. De acordo com Evans (2002) e Lorenzi e Matos (2002), atribuem-se-lhe ainda outros nomes populares, tais como jaborandi-do-paraguai, jaborandi-do-norte, pimenta-de-cachorro e jaborando-manso.

Trata-se de um arbusto (Fig. 1), nativo das regiões sul e sudeste brasileiras, que atinge em média 2-3 $\mathrm{m}$ de altura e é pouco ramificado. Apresenta folhas compostas (Fig. $2,3,5)$, imparipenadas, com folíolos coriáceos com cerca de 8-15 cm de comprimento, oblongo-lanceolados e com ápice emarginado (reentrante) (Fig. 5). As inflorescências (Fig. 3, 4) formam espigas longas, terminais ou axilares, de flores amarelas e pequenas (LORENZI; MATOS, 2002).

As espécies P. jaborandi Holmes, P. microphyllus Stapf ex Wardleworth e $P$. trachylophus Holmes são consideradas correlatas, em razão da composição química semelhante. Estudos fitoquímicos demonstraram a ocorrência de alcaloides, representados principalmente pela pilocarpina [1], e de óleo essencial, cuja composição revela a presença de monoterpenoides (limoneno [2], a-pineno [3], sabieno) e sesquiterpenoides (cariofileno [4]).<smiles>CC[C@H]1COC(=O)[C@@H]1Cc1cncn1C</smiles>

[1]<smiles>C=C(C)[C@H]1CC=C(C)CC1</smiles>

[2]

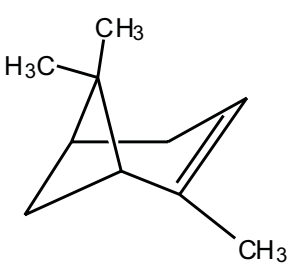

[3]

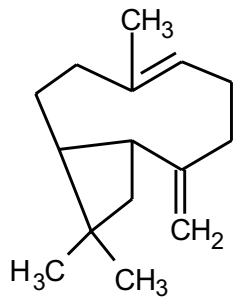

[4]

A pilocarpina [1] é um alcaloide imidazólico, cuja atividade colinérgica tem emprego em oftalmologia. Colírios à base desse alcaloide produzem miose, devido à contração do esfíncter da pupila, e favorecem o escoamento do humor aquoso, diminuindo a pressão intraocular que se encontra aumentada em casos de glaucoma (EVANS, 2002). 
Este trabalho trata do estudo anatômico de folhas de $P$. pennatifolius, com o objetivo de apresentar de maneira didática ilustrações dos caracteres foliares dessa planta medicinal, facilitando o seu reconhecimento e, portanto, contribuindo na execução do controle de qualidade farmacognóstico.

\section{MATERIAL E MÉTODOS}

Coletou-se o material vegetal no Arboreto do Jardim Botânico Municipal de Curitiba, Paraná, localizado aproximadamente na latitude de $25^{\circ} 26^{\prime} \mathrm{S}$, longitude de $49^{\circ} 14^{\prime}$ 'W e altitude de $930 \mathrm{~m}$, em setembro de 2008. A identificação taxonômica correspondeu à exsicata registrada como MBM 311965.

Folhas desenvolvidas foram fixadas em FAA 70 (JOHANSEN, 1940) e posteriormente armazenadas em etanol a 70\% (BERLYN; MIKSCHE, 1976). Estudaramse o terço inferior dos folíolos, incluindo-se as regiões nervural central e internervural, e o peciólulo. Foram feitos cortes à mão livre, nos sentidos paradérmico, transversal e longitudinal, corados com azul de astra e fucsina básica (ROESER, 1972). Paralelamente, o material foi desidratado em série etanólica crescente, emblocado em glicol-metacrilato (Leica Historesin) e seccionado em micrótomo rotativo (KRAUS; ARDUIN, 1997). As estruturas anatômicas foram fotografadas no microscópio de luz Olympus BX40.

\section{RESULTADOS E DISCUSSÃO}

O folíolo, em vista frontal, apresenta células epidérmicas com paredes anticlinais poligonais, moderadamente espessadas, em ambas as faces (Fig. 7, 8). Uma cutícula estriada recobre a epiderme (Fig. 6). Estômatos anomocíticos ocorrem na superfície abaxial (Fig. 8), caracterizando a folha como hipoestomática. As células-guarda se inserem no mesmo nível das demais células epidérmicas e mostram cristas cuticulares externas evidentes (Fig. 11).

Tricomas tectores unicelulares (Fig. 10), de paredes espessadas (Fig. 11) e ápice agudo, bem como alguns tricomas glandulares capitados (Fig. 9), com pedicelo unicelular e glândula pluricelular e plurisseriada estão presentes em ambas as superfícies.

Em secção transversal, observa-se que a epiderme é monosseriada (Fig. 13-15) e o mesofilo é dorsiventral (Fig. 13). Este é constituído de uma camada de parênquima paliçádico (Fig. 12-14) e vários estratos de parênquima esponjoso (Fig. 13-15), que corresponde a $75 \%$ da altura do mesofilo. Feixes vasculares colaterais, de pequeno e médio porte, distribuem-se no parênquima esponjoso e podem apresentar calotas esclerenquimáticas apostas ao floema e ao xilema (Fig. 13,14).

Drusas de oxalato de cálcio são encontradas nos clorênquimas (Fig. 14) e assumem uma disposição caracteristicamente alinhada nas células em paliçada (Fig. 12).

Cavidades secretoras ocorrem no mesofilo (Fig. 12, 15) e na nervura central (Fig. 17). Esta possui secção transversal biconvexa, com curvatura mais evidente junto à superfície abaxial (Fig. 16). Há um feixe vascular colateral, em arranjo cêntrico e envolto por uma bainha esclerenquimática praticamente completa (Fig. 16, 17). O peciólulo mostra-se semelhante à nervura central (Fig. 18-20). 
As monografias farmacopeicas que incluem $P$. pennatifolius, juntamente com $P$. jaborandi e P. microphyllus, sob a denominação comum de jaborandi, estão presentes na F. Bras. II (1959) e F. Bras. III (1977). Ambas edições apresentam transcrições anatômicas idênticas e estas se referem às três espécies de jaborandi, não sendo mencionadas diferenças microscópicas entre elas.

Os resultados observados neste trabalho assemelham-se às descrições anatômicas farmacopeicas, sendo que as ilustrações nele apresentadas permitem a identificação com facilidade dos caracteres indicados, tais como: epiderme com células poligonais em vista frontal, cutícula estriada, tricomas tectores e glandulares, mesofilo dorsiventral, drusas de oxalato de cálcio e cavidades secretoras.

Adicionalmente, essas estruturas secretoras internas são classificadas como cavidades esquizolisígenas nas monografias, embora neste estudo tal conclusão não tenha sido feita, em razão da impossibilidade da execução de estudos ontogenéticos, que permitiriam o acompanhamento do desenvolvimento dessa estrutura foliar.

Com relação aos estômatos, as monografias os descrevem como pequenos e só encontrados na face inferior apenas em grande número. Tal redação pode remeter à ideia de estarem também presentes na superfície superior, próprio de folha anfiestomática. Nesta investigação, determinou-se o tipo de estômato predominante (anomocítico) e, diferindo dessas monografias farmacopeicas, esses anexos epidérmicos foram verificados exclusivamente na face abaxial, caracterizando a folha como hipoestomática.

Na F. Bras. IV (1988-2002), a qual define a droga jaborandi como constituída de folhas ou folíolos de P. microphyllus, sem incluir outras espécies da família Rutaceae, alguns caracteres anatômicos revelam-se diferentes em relação à espécie desta análise. A nervura mediana é mais proeminente na face adaxial e o feixe vascular é contornado por anel esclerenquimático descontínuo em $P$. microphyllus. De modo diverso, a curvatura da nervura central é mais evidente na superfície abaxial e ocorre uma bainha esclerenquimática (anel) praticamente completa ao redor do feixe vascular em $P$. pennatifolius. Ainda, tomando-se por base essa edição farmacopeica, os estômatos são classificados como paracíticos e anomocíticos e de ocorrência exclusiva na face abaxial, o que demonstra correspondência com P. pennatifolius. Na F. Bras. IV (19882002), as cavidades secretoras são também descritas como esquizolisígenas.

Na publicação de Jackson e Snowdon (1990), que trata de uma sucinta descrição de folhas pulverizadas de $P$. microphyllus acompanhada de uma prancha de desenhos, a folha é apresentada como hipoestomática, ocorrendo estômatos anomocíticos, ladeados por 4-6 células pequenas. As demais características também mostram-se semelhantes ao verificado neste estudo, bem como no trabalho de Oliveira e Akisue (1984).

\section{REFERÊNCIAS}

BERLYN, G. P.; MIKSCHE, J. P. Botanical microtechnique and cytochemistry. Ames: lowa State University Press, 1976. 
DUARTE, M. R. Ilustração de caracteres microscópicos de drogas vegetais para o controle de qualidade farmacognóstico. I. Peumus boldus Molina e Mikania glomerata Spreng. Visão Acad., Curitiba, v. 8, p. 5-10, 2007a.

DUARTE, M. R. llustração de caracteres microscópicos de drogas vegetais para o controle de qualidade farmacognóstico. II. Echinodorus macrophyllus e Ruta graveolens. Visão Acad., Curitiba, v. 8, p. 5-10, 2007b.

DUARTE, M. R.; GOLAMBIUK, G. Ilustração de caracteres microscópicos de drogas vegetais para o controle de qualidade farmacognóstico. III. Erva-mate (Ilex paraguariensis A. St.-Hil., Aquifoliaceae). Visão Acad., Curitiba, v. 9, n. 1, p. 5-9, 2008.

EVANS, W. C. Trease and Evans' pharmacognosy. 15th ed. New York: Saunders, 2002.

F. Bras. III - Farmacopéia Brasileira. 3 ed. São Paulo: Andrei, 1977.

F. Bras. IV - Farmacopéia Brasileira. 4 ed. São Paulo: Atheneu, 1988-2002.

F. Bras. II - Farmacopéia dos Estados Unidos do Brasil. 2 ed. São Paulo: Siqueira, 1959.

JACKSON, B. P.; SNOWDON, D. W. Atlas of microscopy of medicinal plants, culinary herbs and spices. London: Belhaven Press, 1990.

JOHANSEN, D. A. Plant microtechnique. New York: McGraw-Hill Book, 1940.

KRAUS, J. E.; ARDUIN, M. Manual básico de métodos em morfologia vegetal. Rio de Janeiro: Edur, 1997.

LORENZI, H.; MATOS, F. J. A. Plantas medicinais do Brasil: nativas e exóticas. Nova Odessa: Plantarum, 2002.

OLIVEIRA, F.; AKISUE, G. Farmacobotânica - curso de identificação de drogas vegetais. São Paulo: Universidade de São Paulo, 1984.

ROESER, K. R. Die Nadel der Schwarzkiefer-Massenprodukt und Kunstwerk der Natur. Mikrokosmos, Stuttgart, v. 61, p. 33-6, 1972. 

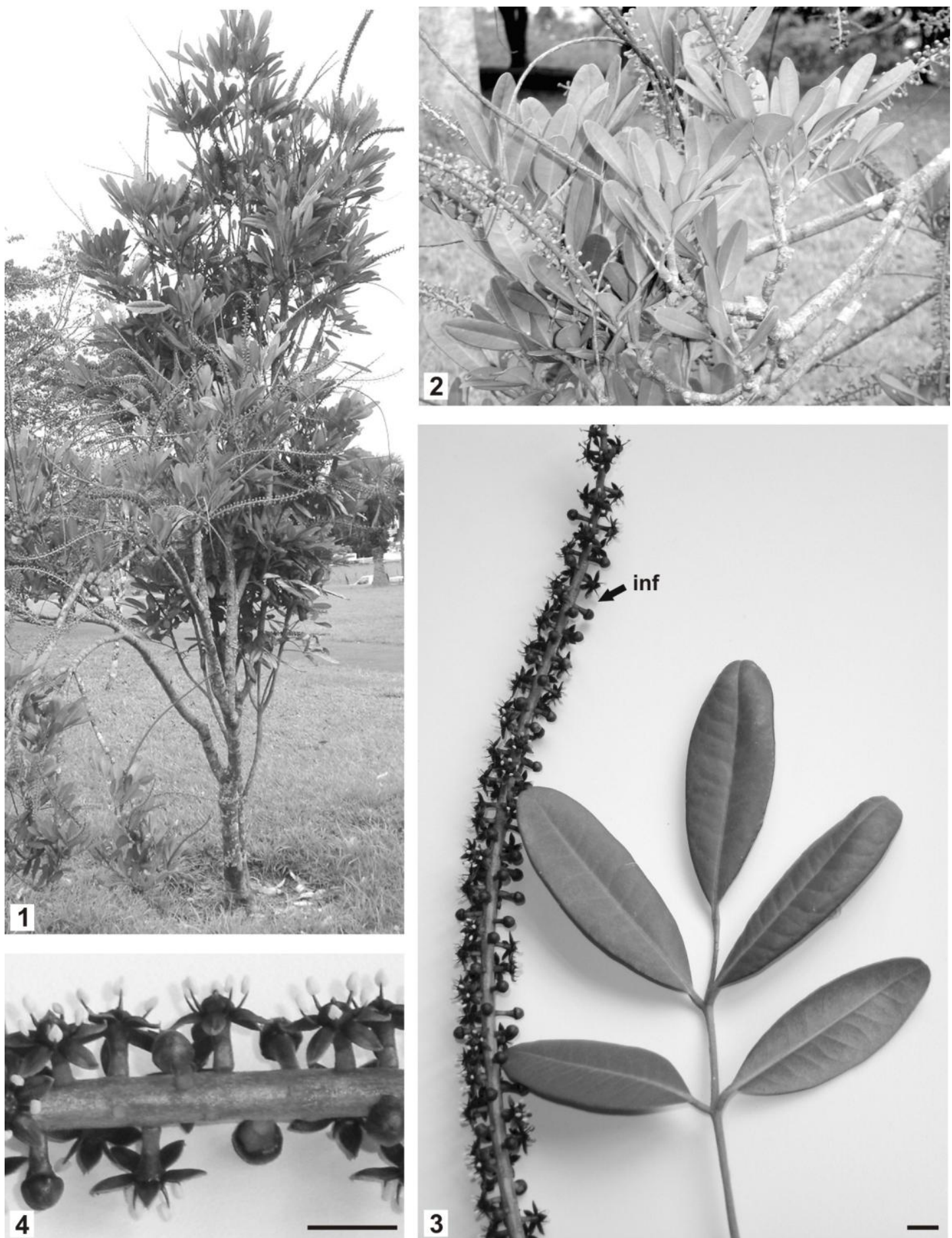

FIGURAS 1-4. JABORANDI (Pilocarpus pennatifolius LEM., RUTACEAE): 1. ASPECTO GERAL DA PLANTA NO HÁBITO; 2. RAMOS VEGETATIVOS E REPRODUTIVOS; 3. INFLORESCÊNCIA E FOLHA COMPOSTA 4. DETALHE DA INFLORESCÊNCIA. ABREVIATURAS: FO - FOLHA COMPOSTA, INF - INFLORESCÊNCIA. BARRA $=1 \mathrm{~cm}(3,4)$ 

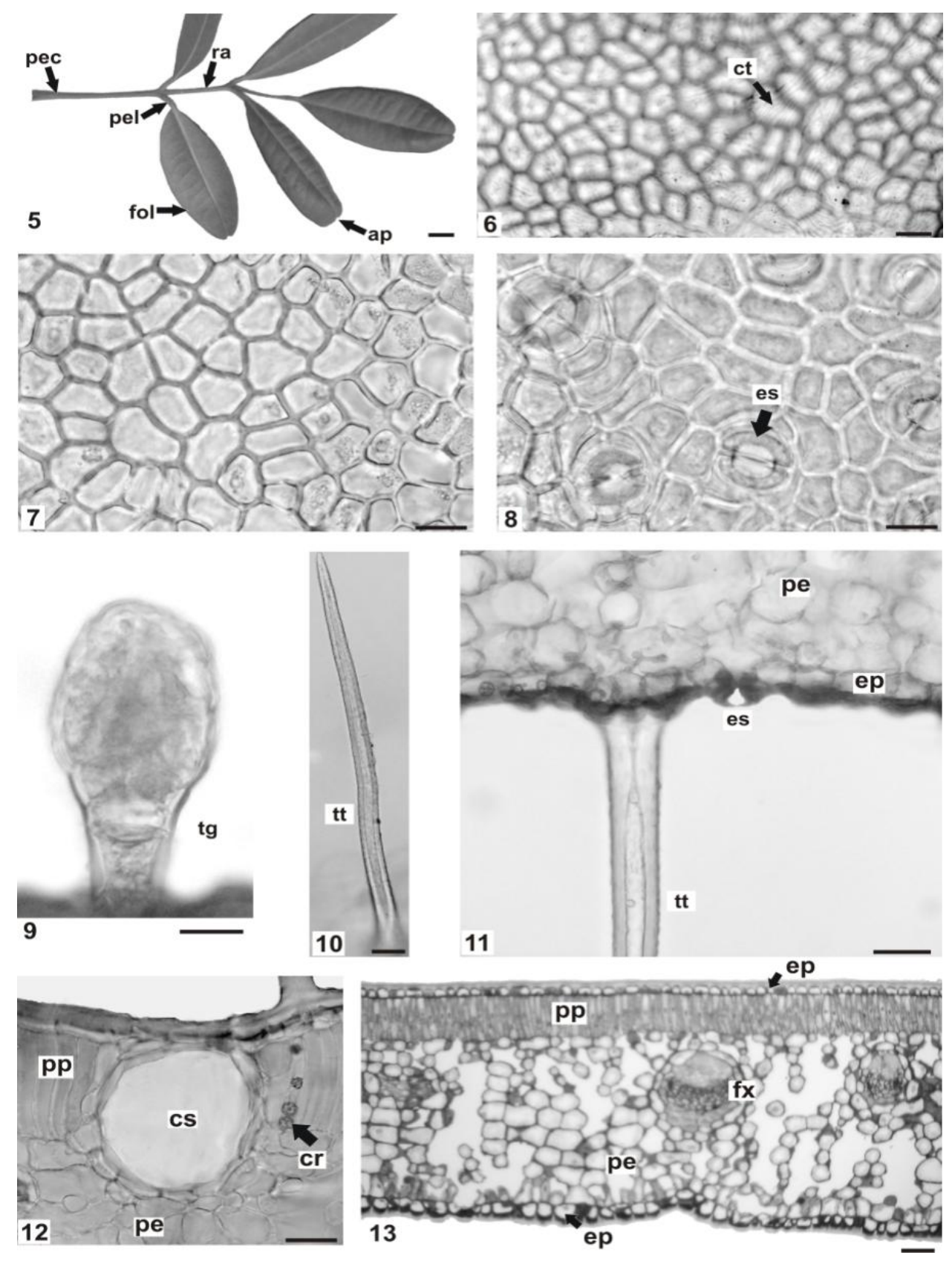

FIGURAS 5-13. JABORANDI (Pilocarpus pennatifolius LEM., RUTACEAE): 5. FOLHA COMPOSTA IMPARIPENADA, EM QUE SE NOTA ÁPICE EMARGINADO (REENTRANTE); 6. VISTA FRONTAL DA EPIDERME FOLIAR, MOSTRANDO CUTÍCULA ESTRIADA; 7, 8. VISTA FRONTAL DA EPIDERME FOLIAR, FACES ADAXIAL E ABAXIAL, RESPECTIVAMENTE. 9. TRICOMA GLANDULAR CAPITADO; 10. TRICOMA TECTOR; 11. PORMENOR DE ESTÔMATO E BASE DE TRICOMA TECTOR; 12. CAVIDADE SECRETORA E DRUSAS NO PARÊNQUIMA PALIÇÁDICO; 13. SECÇÃO TRANSVERSAL DA REGIÃO INTERNERVURAL DO FOLÍOLO, INDICANDO MESOFILO DORSIVENTRAL. ABREVIATURAS: AP - ÁPICE EMARGINADO, CR - CRISTAL DE OXALATO DE CÁLCIO, CS - CAVIDADE SECRETORA, CT - CUTÍCULA, EP - EPIDERME, ES ESTÔMATO, FOL - FOLÍOLO, FX - FEIXE VASCULAR, PE - PARÊNQUIMA ESPONJOSO, PEC PECÍOLO, PEL - PECIÓLULO, PP - PARÊNQUIMA PALIÇÁDICO, RA - RAQUE, TG - TRICOMA GLANDULAR, TT - TRICOMA TECTOR. BARRA $=1 \mathrm{~cm}(5), 50 \mu \mathrm{m}(13), 20 \mu \mathrm{m}(6-12)$ 

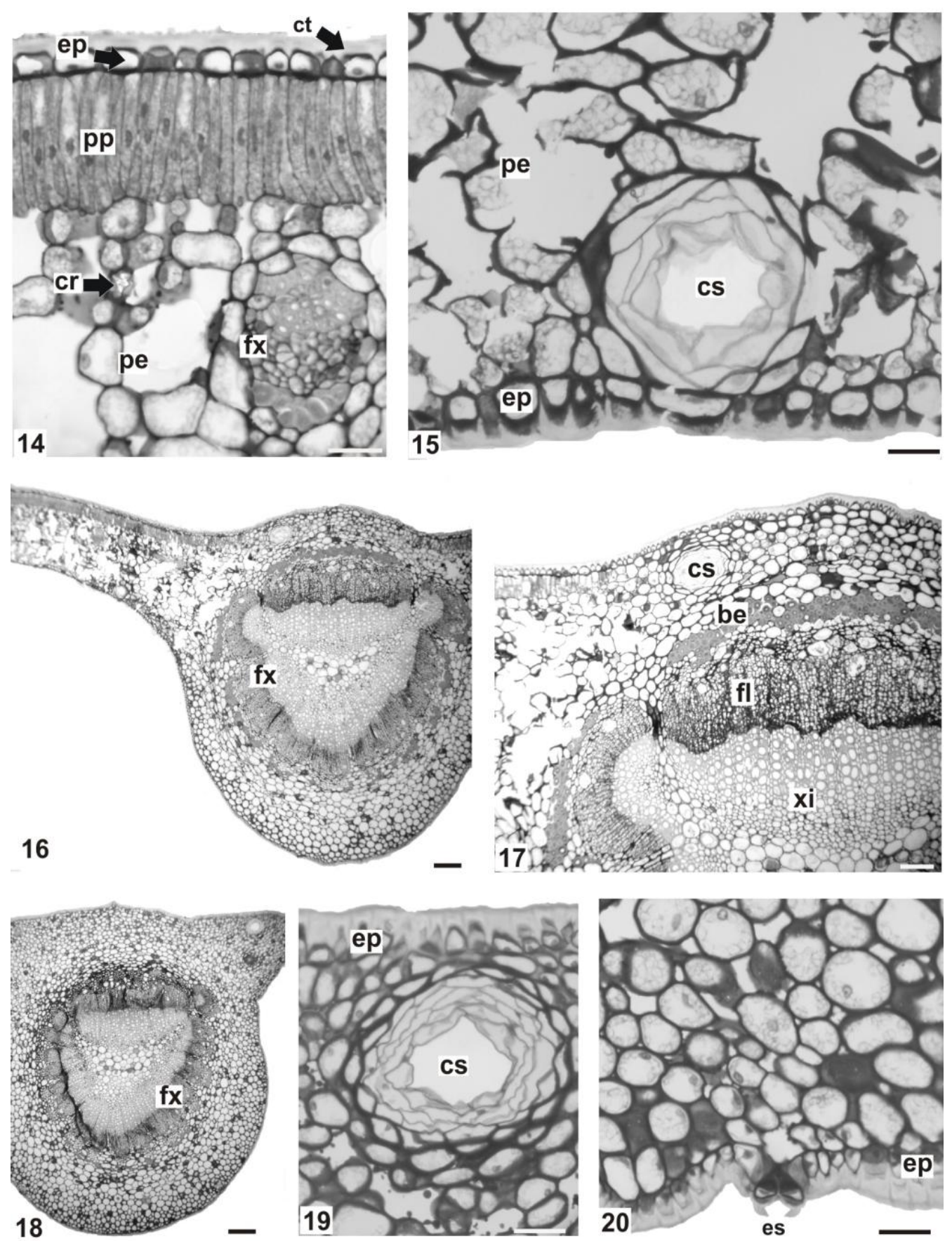

FIGURAS 14-20. JABORANDI (Pilocarpus pennatifolius LEM., RUTACEAE): 14. PORMENOR DE FEIXE VASCULAR DE MÉDIO PORTE EM MEIO AO MESOFILO; 15. CAVIDADE SECRETORA NO PARÊNQUIMA ESPONJOSO; 16. SECCCÃO TRANSVERSAL DA NERVURA CENTRAL, EM QUE SE OBSERVA UM FEIXE VASCULAR; 17. DETALHE DO FEIXE VASCULAR COLATERAL; 18. SECÇÃO TRANSVERSAL DO PECIÓLULO; 19. CAVIDADE SECRETORA NO PECIÓLULO; 20. PORMENOR DE ESTÔMATO NO PECIÓLULO. ABREVIATURAS: BE - BAINHA ESCLERENQUIMÁTICA, CR - CRISTAL DE OXALATO DE CÁLCIO, CS - CAVIDADE SECRETORA, CT - CUTÍCULA, EP - EPIDERME, ES - ESTÔMATO, FL - FLOEMA, FX - FEIXE VASCULAR, PE - PARÊNQUIMA ESPONJOSO, PP - PARÊNQUIMA PALIÇÁDICO, XI XILEMA. BARRA $=100 \mu \mathrm{m}(16,18), 50 \mu \mathrm{m}(17), 20 \mu \mathrm{m}(14,15,19,20)$ 